\title{
Effects of Music Therapy on Surgical and Cancer Patients
}

\author{
Tom Kenyon
}

Orcas, WA, USA

\section{Introduction}

Clinicians dealing with cancer treatments in general, and breast cancer in particular, report a wide range of difficultto-manage responses in their patients including such things as chronic pain, sleep disturbances, stress, anxiety, and depression. Such responses are based upon the severity of prognosis and the unique mental/emotional state of each patient.

Therapists who employ the use of music therapy with patients in a medical setting report that this form of therapy can be highly effective at helping patients reduce the need for pain medications while decreasing depression, stress and anxiety levels. In the next section of this paper, we will explore 7 studies, which would seem to substantiate these claims. While they do not deal with breast cancer specifically, they do address the needs of oncology patients in general.

\section{Studies on the Efficacy of Music Therapy}

Researchers at Bayreuth University, Germany, investigated the effects of music therapy upon heart rate in 28 cancer patients suffering from chronic pain [1]. All subjects were in a stable phase of their cancer. The intervention included a 14day training in relaxation therapy that focused upon falling asleep. Part of this training included a 30-minute lullaby-like music with a gradually decreasing tempo. The researchers compared heart rates on day 1 and 15, focusing upon an analysis of the degree of synchronization, reports by subjects on how long it took to fall asleep, as well as subjective reports on the relaxation therapy and levels of perceived pain. It was discovered that the lullaby-like music had a profound effect. The patients in the relaxation group showed an increasing synchronization and co-ordination of heart rate and musical beat. At a tempo of between 42 and 48 beats per minute, a very sta- ble 2:3 synchronization occurred. Those patients who reported the best relaxation and analgesic effects showed the most synchronization. In this case, music therapy led to an improvement of patients' abilities to fall asleep and to a decrease in their consumption of analgesics.

Researchers at Stanford University School of Medicine, USA, monitored 30 older adults diagnosed with mild to major depressive disorder [2]. Participants were randomly assigned to one of 3 groups. The study was conducted for a period of 8 weeks. Group 1 was given a home-based program where participants learned music listening skills as well as stress reduction techniques through weekly home visits by a music therapist. Group 2 was given the same self-administered program as group 1, but with moderate therapist intervention involving a weekly telephone call. Group 3 was put on a waiting list and used as a control. At the end of the study, participants in groups 1 and 2 performed significantly better than the control group on standardized psychological tests for depression, distress, self-esteem, and mood. These improvements were clinically and statistically significant, and researchers also noted that the benefits gained in groups 1 and 2 (the musical groups) were maintained over a 9-month follow-up period. In a US study of 25 elderly people with self-reported sleep problems, all participants were given classical and New Age music to listen prior to bedtime whenever a disturbance to sleep was identified [3]. Participants were asked to keep daily journals to record the efficacy of music for the induction of sleep. When results were analyzed, researchers found that $96 \%$ of participants (24 out of 25 ) reported improved sleep after listening to music. There were no controls in this pilot study.

Responses in the adrenal cortex to the stress of receiving news about an upcoming surgery to be conducted the next day were studied in a group of 34 patients by monitoring salivary cortisol levels [4]. 18 patients in the study selected a one-hour

\begin{tabular}{ll}
\hline KARGER & ( ) 2007 S. Karger GmbH, Freiburg \\
Fax +497614520714 & Accessible online at: \\
$\begin{array}{l}\text { E-mail Information@Karger.de } \\
\text { www.karger.com }\end{array}$ & www.karger.com/brc
\end{tabular}


music program of their choice, which they listened to immediately following receipt of the information about surgery. The 16 remaining patients received no musical therapy; 10 patients, not awaiting surgery, were the control group. Saliva samples were taken before the stressor and 5 more times at 15-minute intervals each. The initial stress generated a 50\% rise in salivary cortisol within 15 minutes. While the cortisol levels of patients not exposed to music gradually decreased, after one hour, salivary cortisol levels were still markedly higher than the initial level (before the stressor). Those patients who received music therapy exhibited a marked decrease in salivary cortisol, and after one hour, the relative decreases in cortisol levels were similar to those observed in the control group (nonsurgical patients). The results of this study show that music therapy can have a significant positive effect on patients receiving distressing news about required surgery. Researchers suggest that those in authority may wish to consider providing relaxing music to patients in hospitals and health clinics.

The research study of Winter et al. [5] focused on the use of music therapy to reduce anxiety in patients who had chosen elective gynecological surgeries. There were 40 persons in the study with a mean average age of 37 years. All participants were given a physical status classification of either 1 or 2 based upon the American Society of Anesthesiologists guidelines. 31 of the participants listened to prerecorded music while waiting in the surgical holding area. The control group of 19 did not listen to music while waiting in the same surgical holding area. Patient anxiety and stress levels were measured using the STAI (State-Trait Anxiety Inventory), which distinguishes between anxiety that occurs in response to stressful situations (state anxiety) or that, which occurs as a result of personality traits (trait anxiety). Participants in both groups completed the STAI upon entering the surgical holding area and just before being transported to the operating room. Investigators also measured participants' blood pressure and heart rate at both intervals (upon entering and leaving the holding area). The 31 participants who listened to the prerecorded music had significantly lower STAI scores than those who did not listen to music ( $\mathrm{p} \leq 0.05$ ). The mean state anxiety of those participants who listened to the prerecorded music decreased significantly in the period from admission to the preoperative holding area to departure for surgery. However, the anxiety scores of those participants who did not listen to music increased significantly during the time in the holding area. There was no evident correlation between the type of music being played (e.g. classical, country, pop) and reduction in anxiety scores. There were also no appreciable differences between the two groups in terms of blood pressure and heart rate.

Researchers at the Day Surgery Unit of St. Mary's Hospital, Mequon, Wisconsin, USA, conducted a study with 42 ambulatory surgery patients [6]. Half the group listened to music of their choice prior to surgery along with the standard preoperative instructions. The other half received standard preopera- tive instructions without music therapy. Blood pressure, heart rates, and respiratory rates were used as measurements of anxiety and physical stress. Patients in the experimental group presented significantly lower heart rates compared to those in the control group. The experimental group also presented greater improvements in both blood pressure and respiration rates. Researchers concluded that music therapy offers demonstrable benefits for ambulatory surgery patients, and recommended that patients be offered music as an effective option to help alleviate symptoms associated with preoperative anxiety.

Clinicians are well aware that patient anxiety can, and often does negatively affect a wide range of medical procedures. One procedure in particular, sigmoidoscopy, can produce patient anxiety due to the fact that it is complicated and prolonged. In some cases, the procedure has to be abandoned due to patient anxiety. Researchers at Cherry Point Naval Hospital, North Carolina, USA, wanted to see if music therapy might provide a non-drug way to reduce patient anxiety in the face of sigmoidoscopy [7]. 50 adults scheduled for outpatient sigmoidoscopy were divided into two groups. The control group received standard sigmoidoscopy protocol, but no music therapy. The experimental group received standard sigmoidoscopy protocol along with listening to music of their choice during the actual procedure. STAI tests were administered to all subjects before and after the procedures. Physiological recordings of heart rate and mean arterial pressure were also recorded before and during the procedures. Results of the study showed that those patients who listened to self-selected music during the procedure had significantly decreased STAI scores. Their heart rates and arterial pressures were also decreased compared to the control group. Researchers concluded that music is an effective anxiolytic, which can offer significant benefits to patients having to undergo medical procedures such as flexible sigmoidoscopy. In their concluding remarks, the researchers stated: 'Music therapy is an easily administered, inexpensive, safe and effective nonpharmaceutical anxiolytic for patients undergoing flexible sigmoidoscopy.... Music therapy could be a useful adjunct to reduce anxiety in a variety of other diagnostic as well as therapeutic colorectal procedures.'

While the above studies do not focus on breast cancer patients in particular, some of the described problems are similar to those faced by surgical and cancer patients in general. The studies would seem to support what music therapists working in clinical settings with breast cancer patients report in general - namely that music can help these patients cope better with their treatments by reducing stress, anxiety, depression, and the need for analgesics.

\section{Musical Tempo in Music Therapy}

Researchers at the Lozanov Institute in Bulgaria were some of the first to document that musical tempo affected EEG activi- 
ty, heart rate, blood pressure, respiration, and galvanic skin response. One of their first studies involved Bach's 'Goldberg Variations', a Baroque composition for harpsichord. Bach had a good friend, named Count Kayserling, who was a Russian envoy. The diplomat evidently suffered from insomnia and asked Bach if he might compose something that his (Kayserling's) personal harpsichordist could play for him when he had trouble sleeping. Bach named the composition 'The Goldberg Variations'. Dr. Lozanov noted that subjects listening to the 'Variations' experienced an increase of alpha activity, a known precursor to the slow wave activity of sleep. In fact, the 'Variations' increased alpha activity by an average of $6 \%$, while decreasing beta activity (alertness) also by an average of $6 \%$. As Lozanov studied the relationships between tempo and brain entrainment further, he discovered that music at a tempo of around 60 beats per minute increased alpha activity. He further discovered that the largo movements of Baroque music all produced similar effects.

In the study at Bayreuth University, cited at the beginning of this paper [1], researchers noted that music at a tempo of between 42 and 48 beats per minute produced a very stable 2:3 synchronization in heart rhythm and that such synchronizations seemed to correspond with decreases in the need for analgesics.

Such studies and reports of music therapists in general show that the alteration of musical tempo is one of the primary agents involved in the relaxation response and stress-reducing abilities of music. Specifically, tempos between 60 and approx. 42 beats per minute seem to have maximum effect in this regard.

\section{Psychological Aspects of Music Therapy}

While it is not in the scope of this paper to discuss the numerous psychological effects of music therapy, the author considers it vital for clinicians using music therapy to be sensitive to these effects.

We would be remiss if we concluded that the results of music therapy will always be the same for all patients. Each patient is unique, with personal experiences that are generated out of his or her life experience, beliefs, and medical situation. Music therapy can have a profound effect upon the mental and emotional experiences of patients due to the fact that it alters neurological events in the brain and nervous system. For instance, as EEG activity enters into the lower states of alpha and/or theta, there is a decrease of stress and tension. However, under some conditions, subconscious psychological material may also surface into conscious awareness. This can be a very dynamic process for those facing life-threatening or protracted illnesses.

For most cases, relaxing-type music, as described in the aforementioned studies, will produce classic signs of relaxation with its attendant decreases in anxiety, stress, and the need for analgesics. For some patients, however, this same type of music can bring into conscious awareness difficult emotions as well as challenging personal and interpersonal issues. In most cases, a friendly ear is all that is needed - someone to listen without judgment, someone to simply be a witness.

In addition, patients facing life-threatening illnesses sometimes report transpersonal (spiritual) experiences as a result of being exposed to music therapy. These types of experiences are generated out of a patient's own belief system, expectation, and the possibility or inevitability of death. Patients unfamiliar with 'spiritual' experiences may be confused or reluctant to talk about them. Clinicians are encouraged to handle transpersonal experiences in their patients in a similar way as they would psychological issues. The patient often simply needs to have someone listen. In some cases, if the experiences are too outside the 'norm' of a patient's expectation, he/she may need to be reassured that he/she is not 'crazy' and that such experiences, though unusual, are natural when confronted with a major life change. Clinicians are encouraged to listen to their patients without imposing their own personal beliefs. Transpersonal experiences that arise from music therapy often leave a patient feeling particularly sensitive and vulnerable, and caregivers need to be aware of this.

\section{Final Suggestions for Clinicians}

Music therapy can be roughly divided into two categories active and passive. In active forms of music therapy, patients play music, sing or are, in some way, encouraged to create or describe their experiences with music. Passive forms of music therapy involve the patient simply listening to either 'live' or prerecorded music. In all of the studies cited in this paper, patients were exposed to passive forms of therapy. This type of music therapy can easily be introduced into clinical situations involving a minimum of equipment and/or staff attention. Simply having a patient listen to relaxing music of his choice, can have profound effects upon stress and anxiety levels, and a relaxed patient is more easily managed than one who is overly anxious and stressed.

Music therapists often report a critical element in passive forms of listening - the patient must like the music. Therefore, clinicians desiring to create a music therapy program for their patients in a clinical setting should have a wide selection of relaxing music to choose from. Some simple personal listening devices with headphones are all that is needed. Patients should be encouraged to listen to music of their own choosing.

Generally speaking, largo movements from Classical and Baroque music will generate the desired state of relaxation. In addition, some modern and/or New Age music can be highly effective as well. Finally, a new form of therapeutic sound, known as psychoacoustic music, can be highly effective at producing relaxed states of body and mind. The critical 
elements are a slow repetitive rhythm, a soothing timbre (tone quality), and no words. The exception to this would be repetitive chants in a nonnative language that the listener would not recognize.

Breast cancer patients undergoing chemotherapy are ideally suited for music therapy, in that during chemotherapy there is nothing for them to do. In addition to reading materials, the clinician can offer his patients the option of listening to therapeutic music during their treatments. This is an ideal clinical opportunity for patients to experience the numerous benefits of music therapy.
For those clinicians who do not have access to the input of a professional music therapist, in addition to the above-named elements, there are two other possibilities that will help determine what forms of music should be used. 1 . The clinician's own response - if a recording makes him/her feel relaxed, chances are it will have a similar effect on others. 2. Patient feedback - the clinician can ask his/her patients to comment on the music he/she has chosen and to state what types of music make them feel the most relaxed. The clinician can encourage them to share their favorite relaxation music with other patients by writing the titles in a Music Suggestions Log.

\section{References}

1 Reinhardt U: Untersuchungen zur Synchronisation von Herzfrequenz und musikalischem Rhythmus im Rahmen einer Entspannungstherapie bei Patienten mit tumorbedingten Schmerzen. [Investigations into synchronisation of heart rate and musical rhythm in a relaxation therapy in patients with cancer pain.] Forsch Komplementärmed 1999;6: 135-141.

2 Hanser SB, Thompson LW: Effects of a music therapy strategy on depressed older adults. J Gerontol 1994;49:265-269.
3 Mornhinweg GC, Voignier RR: Music for sleep disturbance in the elderly. J Holist Nurs 1995;13: $248-254$.

4 Miluk-Kolasa B, Obminski Z, Stupnicki R, Golec L: Effects of music treatment on salivary cortisol in patients exposed to pre-surgical stress. Exp Clin Endocrinol 1994;102:118-220.

5 Winter MJ, Paskin S, Baker T: Music reduces stress and anxiety of patients in the surgical holding area. J Post Anesth Nurs 1994;9:340-343.
6 Augustin P, Hains AA: Effect of music on ambulatory surgery patients' preoperative anxiety. AORN J 1996;63:750.

7 Palakanis KC, De Nobile JW, Sweeney WB, Blankenship CL: Effect of music therapy on state anxiety in patients undergoing flexible sigmoidoscopy. Dis Colon Rectum 1994;37:478-481. 